\title{
Discrete Version of Richard's Theorem and Applications to Cascaded Lattice Realization of Digital Filter Transfer Matrices and Functions
}

\author{
P. P. VAIDYANATHAN, MEMBER, IEEE, AND SANJIT K. MITRA, FELLOW, IEEE
}

\begin{abstract}
The well-known Richards' Theorem of the continuous-time filter theory is reformulated in the digital domain in a convenient manner, leading to a simple derivation of cascaded lattice digital filter structures, realizing lossless bounded transfer functions. The theorem is also extended to the matrix case, leading to a derivation of $m$-input $p$-output cascaded lattice filter structures with lossless building blocks, that realize an arbitrary $p \times m$ digital Lossless Bounded Real (LBR) transfer matrix. Extensions to the synthesis of arbitrary, stable $p \times m$ transfer matrices in the form of such cascaded lattices is also outlined. The derivation also places in evidence a means of testing the stability of an arbitrary $p \times m$ transfer matrix of a discrete-time linear system.
\end{abstract}

\section{INTRODUCTION}

A $\mathrm{N}$ IMPORTANT result in continuous-time filter theory is the theorem due to Richards [1], [2], which has found applications both in lumped-parameter and distributed passive network synthesis. Basically, it relates a Positive Real (PR) function to another, and indicates a means of degree reduction of an impedance during the synthesis process. To be specific, let $Z(s)$ be a real rational PR function. Define $Z_{1}(s)$ according to

$$
Z_{1}(s)=Z(1) \frac{Z(s)-s Z(1)}{Z(1)-s Z(s)}
$$

Then the theorem asserts that $Z_{1}(s)$ is also a PR function of degree no larger than that of $Z(s)$. Moreover, if $Z(-1)$ $=-Z(1)$, then the degree of $Z_{1}(s)$ is 1 lower than that of $Z(s)$. One of the major applications of this theorem is in the synthesis of distributed networks based on the extraction of unit elements.

A well-known family of structures in digital filter theory is the cascaded-lattice structures [3], [4], studied extensively by Gray and Markel. While several interpretations and derivations of these structures are known (for example, those based on orthogonal polynomial theory [3], prediction theory [5], and digital two-pair extraction theory [6]), it is also known that these structures are related to the unit

Manuscript received October 15, 1984; revised March 22, 1985. This work was supported in part by the National Science Foundation under Grant ECS 82-18310 and under Grant ECS 84-04245.

P. P. Vaidyanathan is with the Department of Electrical Engineering, California Institute of Technology, Pasadena, CA 91125.

S. K. Mitra is with the Department of Electrical and Computer Engineering, University of California, Santa Barbara, CA 93106.

IEEE Log Number 8405846 elements of distributed network theory [7]. It, therefore, seems that, the synthesis of the Gray and Markel structures is related to the Richards' theorem. In this paper, we obtain a new derivation of the cascaded lattice digital filter structures, based on a digital version of Richard's theorem in terms of Bounded Real (BR) functions. In order to make the derivations self-contained, an independent derivation of this version of the Richards' theorem in the discrete-time domain is first given in Section II, and this is followed by the derivation of the lattice structures. In this context, we also wish to draw the attention of the reader to an interesting paper by Delsarte et al. [23] which reveals the relationship of lattice structures and stability test procedures to the classical Nevanlinna-Pick interpolation problem.

In Section III, we state and prove an extended version of the discrete-time counterpart of the Richard's theorem for m-input p-output BR transfer matrices. This is then used to derive new cascaded lattice realizations for $m$-input $p$-output linear systems, characterized by $p \times m$ Lossless Bounded Real (LBR) or "all-pass" transfer matrices. A special case of these structures is reported in [26], based on a different approach for derivation. Such realizations have considerable importance in the synthesis of low-sensitivity digital filter structures. An example of such an application can be found in [22]. Moreover, the extreme suitability of such structures for the VLSI implementation of low-sensitivity digital filters has been recently established [8], [9]. The synthesis proccdure simultaneously reveals how to realize an arbitrary stable $p \times m$ transfer matrix (not necessarily LBR) in the form of a cascaded lattice structure. The derivation also places in evidence a procedure for testing the stability of a $p \times m$ transfer matrix, which is described in Section IV.

Even though the results of Section II are special cases of those in Section III, the derivation in Section II from the Richards' theorem viewpoint reveals the simplicity of the scalar lattice synthesis. The results of Section III also reduce to the special case of certain pipelineable orthogonal digital filters, reported elsewhere [8], [9].

All results are stated and derived directly in the $z$-domain, in terms of dimensionless transfer functions and matrices. It should, however, be noted that, by employing PR (posi- 
tive real) to $\mathrm{BR}$ transformations along with the bilinear transformation, one can obtain some of our results from well-established results of continuous time filter theory. Such an approach, and the concept of impedance functions and matrices are however, not used anywhere in the sequel. We fecl that an independent $z$-domain approach leads to a simplified understanding of the lattice structures, at the same time explicitly showing the relation to continuous-time network theory. Another motivation for this is that, while the scattering matrices of continuous-time lossless multiports [10], [18] do satisfy the LBR property, they are in general square matrices, rather than general $p \times m$ matrices. Thus a straightforward translation of known results of classical filter theory is not convenient for lattice-synthesis of rectangular transfer matrices.

\section{Notations}

In this paper boldfaced letters indicate matrices and vectors, for example, $\mathbf{H}(z), \mathbf{v}(n)$ etc. The tilde-accent stands for transposition followed by reciprocation of functional argument; for example: $\tilde{\mathbf{H}}(z)=\mathbf{H}^{t}\left(z^{-1}\right)$. Superscript $t$ stands for matrix transposition. Superscript dagger $(\dagger)$ stands for transposition followed by complex conjugation. The notation $\mathbf{A} \leqslant \mathbf{B}$ is abbreviation for " $\mathbf{B}-\mathbf{A}$ is positive semi-definite", and $\mathbf{A}<\mathbf{B}$ is abbreviation for " $\mathbf{B}-\mathbf{A}$ is positive definite". $\mathbf{I}_{m}$ stands for the identity matrix of dimension $m \times m$. The symbol $\mathbf{0}$ stands for null vectors and null matrices of appropriate dimensions. For a (real symmetric) positive definite matrix $\mathbf{P}$, we define its square root $\mathbf{P}^{1 / 2}$ according to the factorization: $\mathbf{P}=\mathbf{P}^{1 / 2} \mathbf{P}^{t / 2}$ where $\mathbf{P}^{t / 2}$ stands for the transpose of $\mathbf{P}^{1 / 2}$.

\section{Alternate Derivation of the Scalar Cascaded Lattice Structures}

Let us first review a few basic definitions. A digital filter transfer function $H(z)$ given by

$$
H(z)=\frac{\sum_{n=0}^{N} a_{n} z^{-n}}{\sum_{n=0}^{N} b_{n} z^{-n}}
$$

is said to be Bounded Real (BR) if it is stable, ${ }^{1}$ real-valued for real $z$, and satisfies the boundedness property

$$
\left|H\left(e^{j \omega}\right)\right| \leqslant 1 .
$$

Assuming $b_{0} \neq 0$ and $b_{N} \neq 0$, the integer $N$ is called the degree of the BR function. By employing the maximum modulus theorem of complex-variable theory, it can be shown that

$$
|H(z)| \leqslant 1, \quad|z|>1
$$

with equality holding in (4) for some $z$ only if $H(z)$ is a constant function. A Lossless Bounded Real (LBR) transfer function $H(z)$ is a BR function, with equality holding

\footnotetext{
'In this paper "stability" stands for bounded input bounded outpu stability [11], i.e., all poles are strictly inside the unit circle.
}

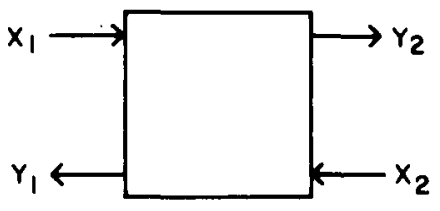

Fig. 1. A digital two-pair.

in (3) for all $\omega$. Such a function is more commonly known as a stable all-pass function. These concepts can be extended to the general m-input p-output linear discrete-time systems in an obvious manner. Thus a $p \times m$ transfer matrix $\mathbf{H}(z)$ is said to be BR if it is stable, real-valued for real $z$ and

$$
\mathbf{H}^{\dagger}\left(e^{j \omega}\right) \mathbf{H}\left(e^{j \omega}\right) \leqslant \mathbf{I}_{m}
$$

for all $\omega$. For an LBR transfer matrix, equality holds in (5) for all $\omega$. For LBR transfer functions and matrices, the following paraunitary property [10]:

$$
\tilde{\mathbf{H}}(z) \mathbf{H}(z)=\mathbf{I}_{m}
$$

holds for all $z$. We wish to note here that, for an LBR transfer matrix, $p$ has to be at least as large as $m$. For, if $p$ were smaller than $m$, the rank of the matrix $\mathbf{H}^{\dagger}\left(e^{j \omega}\right) \mathbf{H}\left(e^{j \omega}\right)$ cannot exceed $p$, and the matrix cannot be equal to $\mathbf{I}_{m}$. Note also that, scattering matrices of continuous-time lossless multiports satisfy the LBR property [10], but are square matrices, i.e., $p=m$.

In this paper, we have frequent occasion to deal with a digital two-pair [6]. Such a system (Fig. 1) is a two-input two-output system, described either by the chain parameters, $A(z), B(z), C(z), D(z)$ :

$$
\left[\begin{array}{l}
X_{1}(z) \\
Y_{1}(z)
\end{array}\right]=\left[\begin{array}{ll}
A(z) & B(z) \\
C(z) & D(z)
\end{array}\right]\left[\begin{array}{l}
Y_{2}(z) \\
X_{2}(z)
\end{array}\right]
$$

or by the transfer parameters $\left[T_{i j}\right]$ :

$$
\left[\begin{array}{l}
Y_{1}(z) \\
Y_{2}(z)
\end{array}\right]=\left[\begin{array}{ll}
T_{11}(z) & T_{12}(z) \\
T_{21}(z) & T_{22}(z)
\end{array}\right]\left[\begin{array}{l}
X_{1}(z) \\
X_{2}(z)
\end{array}\right] .
$$

The two descriptions are related as

$$
\begin{aligned}
& T_{11}=C / A, \\
& T_{12}=(A D-B C) / A, \\
& T_{21}=1 / A, \\
& T_{22}=-B / A .
\end{aligned}
$$

A digital two-pair is said to be LBR if the transfer matrix of (8) is LBR.

Given a scalar transfer function $H(z)$, let us consider a new transfer function $G(z)$, defined according to

$$
G(z)=\left(\frac{1-a z}{z-a}\right) \frac{H(z)-H(a)}{1-H(z) H(a)} .
$$

Assume that $H(z)$ is not a constant function. It is clear that $(z-a)$ is a factor of the numerator of the quantity $H(z)-H(a)$, so that the apparent "pole" at $z=a$ in (10) cancels. In addition, if the constant $a$ is chosen such that

$$
H(a) H(1 / a)=1
$$


then factor $(1-a z)$ in $(10)$ cancels with a corresponding factor in $[1-H(z) H(a)]$. This choice of $a$ then ensures that the denominator of the function $G(z)$ is of lower degree than that of $H(z)$.

Let us now assume that $H(z)$ is BR and that $|a|>1$. This ensures the following inequalities:

$$
|H(a)|<1
$$

and

$$
|H(z)| \leqslant 1, \quad|z| \geqslant 1 \text {. }
$$

Consequently, all poles of $G(z)$ are strictly inside the unit circle, and hence $G(z)$ is stable. Furthermore, on the unit circle of the $z$-plane, where we have

$$
\left|\frac{1-a z}{z-a}\right|=1
$$

the following inequality holds:

$$
\left|\frac{H(z)-H(a)}{1-H(z) H(a)}\right| \leqslant 1, \quad|z|=1 .
$$

This follows immediately from (12) and (13). In effect, we have proved the following discrete-time, BR-version of Richards' Theorem:

Theorem 2.1: Let $H(z)$ be a BR function of degree $N$, and let $a$ be any real number such that $|a|>1$. Then the function $G(z)$ defined as in (10) is BR. Moreover, if $a$ is such that (11) holds, then the degree of $G(z)$ is (at most) equal to $N-1$.

Fig. 2 shows a circuit interpretation of (10). In (10), the factor $(1-a z) /(z-a)$ cancels as explained in the text above. In the network of Fig. 2, however, the quantity $(z-a) /(1-a z)$ (rather than $(1-a z) /(z-a))$ appears as a transfer function block. Thus even though (10) involves the cancellation of an unstable pole, the cancellation involved in Fig. 2 is that of a stable pole. We therefore do not have any imperfect cancellation problems due to quantization effects. Note that, in general the structure has a delay free loop. If however, $a$ is chosen to be $\infty$, then the circuit of Fig. 3 results, and has no delay-free loops. This is precisely the "two-multiplier lattice structure of Gray and Markel [12]." The structure of Fig. 3 can be looked upon as a digital two-pair [6], terminated in a constraining transfer function $z^{-1} G(z)$. The two-pair transfer parameters are

$$
\begin{array}{ll}
T_{11}=k, & T_{12}=\left(1-k^{2}\right), \\
T_{21}=1, & T_{22}=-k
\end{array}
$$

or equivalently,

$$
A=1, \quad B=k, \quad C=k, \quad D=1
$$

where

$$
k=H(\infty)
$$

From (7) it is clear that scaling of the chain parameters does not affect $H(z)$ for a given $G(z)$ or vice versa. In particular, if they are scaled by a factor $\sqrt{1-k^{2}}$, it results in a two-pair with transfer parameters:

$$
T_{11}=k, \quad T_{12}=T_{21}=\sqrt{1-k^{2}}, \quad T_{22}=-k
$$

and leads to an interpretation of (10) as shown in Fig. 4.

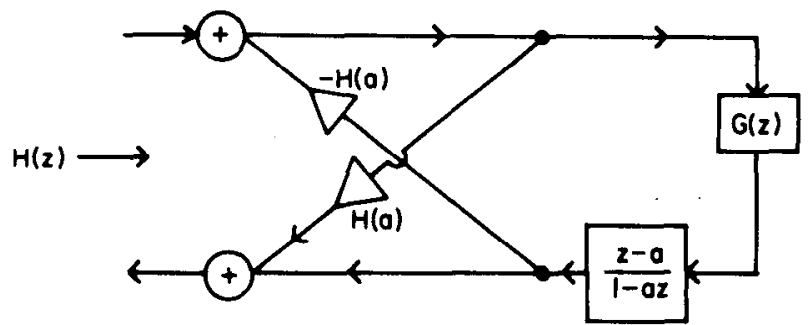

Fig. 2. Circuit representation of (10).

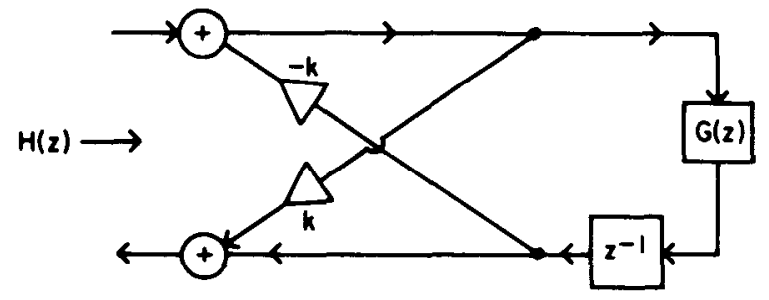

Fig. 3. Special case, where $a=\infty$.

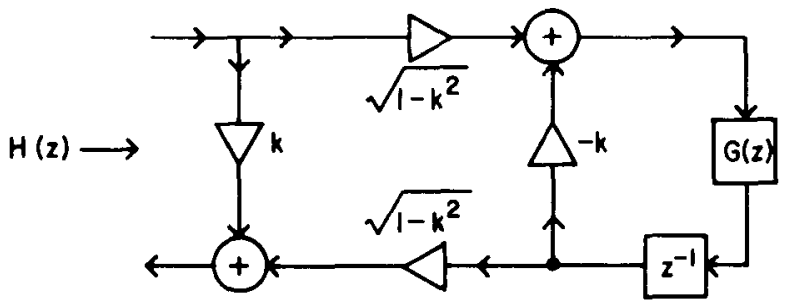

Fig. 4. The normalized circuit.

This is the normalized Gray and Markel lattice structure [4]. In fact, all the five lattice structures given in [12] can be obtained simply by scaling the chain parameters of (17). The structures shown in Figs. (2)-(4) can be interpreted as extracting a digital two-pair from a BR function $H(z)$, leading to a reduced-degree remainder BR function. If this process is repeated, it leads to a complete cascaded lattice realization. The normalized two-pair, described by (19) is easily seen to be LBR.

If the BR function $H(z)$ is actually LBR, then the condition of (11) is always satisfied for all $a$, and a cascaded realization of the above form is always possible. In particular, if $|a|>1$ then the remainder is reduced order BR. In fact the remainder is LBR because, as seen from (10), $\left|G\left(e^{j \omega}\right)\right|$ is unity whenever $\left|H\left(e^{j \omega}\right)\right|$ is unity. The choice $a=\infty$ actually leads to the familiar cascaded-lattice realization of allpass functions [6]. Even though there exist delay-free loops in the structure of Fig. 2 unless $a=\infty$, choice of finite values for $a$ has important applications in procedures for testing the stability of transfer functions, where delay-free loops do not matter. Further elaborations of this point, and an actual numcrical cxample demonstrating it are presented in Section IV.

\section{iII. Extension to the $p \times m$ Transfer Matrix Case}

Let $\mathbf{H}(z)$ represent a m-input p-output digital filter transfer function, given in the form of a right Matrix Fraction Description (MFD) [13], [20]:

$$
\mathbf{H}(z)=\mathbf{N}(z) \mathbf{D}^{-1}(z)
$$


where $\mathbf{N}(z)$ and $\mathbf{D}(z)$ are matrix polynomials of dimensions $p \times m$ and $m \times m$, respectively, as given by

$$
\begin{aligned}
& \mathbf{N}(z)=\mathbf{A}_{0} z^{N}+\mathbf{A}_{1} z^{N-1}+\cdots+\mathbf{A}_{N} \\
& \mathbf{D}(z)=\mathbf{B}_{0} z^{N}+\mathbf{B}_{1} z^{N-1}+\cdots+\mathbf{B}_{N} .
\end{aligned}
$$

$N$ is the degree of the MFD. If the transfer matrix of (20) is BR, then (5) holds, by definition. In addition, an inequality, analogous to (4) holds [26]. Specifically,

$$
\mathbf{H}^{\dagger}(z) \mathbf{H}(z) \leqslant \mathbf{I}_{m}
$$

for all $z$ such that $|z|>1$. Moreover, [26] if there exists a vector $v$ such that, for $|z|>1$ equality holds in (23), i.e., if $\mathbf{v}^{\dagger} \mathbf{H}^{\dagger}(z) \mathbf{H}(z) \mathbf{v}=\mathbf{v}^{\dagger} \mathbf{v}$, then $\mathbf{H}(z) \mathbf{v}$ is constant i.e., independent of $z$. This in turn implies that $\mathbf{H}(z)$ is memoryless in the direction $\mathbf{v}$; in other words, if we apply an input sequence of the form

$$
\mathbf{x}(n)=s(n) \mathbf{v}
$$

to the system $\mathbf{H}(z)$, then the output sequence $\mathbf{y}(n)$ does not depend upon $\mathbf{x}(n-k)$, for $k>0$. Thus (23) holds with strict inequality, unless $\mathbf{H}(z)$ is memoryless in certain directions.

Given a $p \times m$ transfer matrix $\mathbf{H}(z)$, let us now consider a new $p \times m$ transfer matrix $\mathbf{G}(z)$ :

$$
\begin{aligned}
& \mathbf{G}(z)=\left(\frac{1-a z}{z-a}\right)\left(\mathbf{I}_{p}-\kappa \kappa^{t}\right)^{-1 / 2}[\mathbf{H}(z)-\kappa] \\
& \cdot\left[\mathbf{I}_{m}-\kappa^{t} \mathbf{H}(z)\right]^{-1}\left(\mathbf{I}_{m}-\kappa^{t} \kappa\right)^{1 / 2} .
\end{aligned}
$$

Clearly this can be identified to be a right MFD form, similar to (20): i.e.,

$$
\mathbf{G}(z)=\mathbf{N}_{1}(z) \mathbf{D}_{1}^{-1}(z) .
$$

The $p \times m$ matrix $\kappa$ is defined to be

$$
\kappa=\mathbf{H}(a)
$$

where $a$ in $(25)$ is a real scalar.

In the rest of the sequel, we assume that $\mathbf{H}(z)$ is proper, i.e., $\mathbf{H}(\infty)$ is finite. (If $\mathbf{H}(z)$ is causal and stable, this property is automatically satisfied). In addition, we assume (without any loss of generality) that the $m \times m$ matrix $\mathbf{D}(z)$ is column-reduced, i.e., the determinantal degree is equal to the sum of the column degrees [13]:

$$
\operatorname{deg}(\operatorname{det} \mathbf{D}(z))=\sum_{i=1}^{m} k_{i}
$$

where $k_{i}$ is the degree of the $i$ th column ${ }^{2}$ of $\mathbf{D}(z)$. Equation (28) is of course an upper bound on the determinantal degree of a matrix $\mathbf{D}(z)$.

Now from (25) it is clear that, the factor $(z-a)$ cancels with a corresponding factor in $[\mathbf{H}(z)-\kappa]$. Next assume that $a$ is such that the following equality holds:

$$
\left[\mathbf{I}_{m}-\boldsymbol{\kappa}^{t} \mathbf{H}(1 / a)\right]=\mathbf{0} \text {. }
$$

This implies that the quantity $\left[\mathbf{D}(z)-\kappa^{t} \mathbf{N}(z)\right]$ can be written as

$$
\left[\mathbf{D}(z)-\kappa^{t} \mathbf{N}(z)\right]=(1-a z) \mathbf{F}(z)
$$

\footnotetext{
${ }^{2}$ That is, degree of the highest degree polynomial in the $i$ th column of the matrix $\mathbf{D}(z)$.
}

Because of the "properness" of $\mathbf{H}(z)$, the column degree $k_{i}$ of each column of $\mathbf{D}(z)$ is at least as large as the degree of the corresponding column of $\kappa^{t} \mathbf{N}(z)$ [13]. Thus the determinantal degree of the matrix in (30) is bounded above by (28). In other words, the determinantal degree of $F(z)$ is less than (28) by at least $m$. But the right-hand side of (28) is precisely the determinantal degree of $\mathbf{D}(z)$. In conclusion, the choice of $a$ according to (29) leads to a (determinantal) degree reduction by at least $m$ :

$$
\operatorname{deg}\left(\operatorname{det} \mathbf{D}_{1}(z)\right) \leqslant \operatorname{deg}(\operatorname{det} \mathbf{D}(z))-m \text {. }
$$

Let us now assume that $\mathbf{H}(z)$ is BR, and that $|a|>1$. Then $\kappa$ given by (27) satisfies

$$
\boldsymbol{\kappa}^{t} \boldsymbol{\kappa} \leqslant \mathbf{I}_{m}, \quad \boldsymbol{\kappa} \boldsymbol{\kappa}^{t} \leqslant \mathbf{I}_{p} .
$$

Thus the matrices $\left(\mathrm{I}_{p}-\kappa \kappa^{t}\right)$ and $\left(\mathrm{I}_{m}-\kappa^{t} \kappa\right)$ are positive semi-definite (hence the matrix square-roots in (25) are meaningful). Moreover, if $\mathbf{H}(z)$ is not memoryless in any direction, then the inequality (23) is strict, hence the inequalities in (32) are strict, hence $\left(\mathbf{I}_{p}-\kappa \kappa^{t}\right)$ and $\left(\mathbf{I}_{m}-\kappa^{t} \kappa\right)$ are strictly positive definite. This makes the inverses in (25) meaningful. Finally, with $\mathbf{H}(z)$ a BR function, we can show that $\mathbf{G}(z)$ is also a BR function (proof to follow). We can summarize these observations in the following theorem which is thus a generalization of the Richards' theorem, for $p \times m$ discrete-time BR transfer matrices.

Theorem 3.1: Let $\mathbf{H}(z)$ be a $p \times m$ BR transfer matrix, given by the right MFD of (20), with $\mathbf{D}(z)$ column-reduced. Assume that $\mathbf{H}(z)$ is not memoryless in any direction. Let $a$ be a real scalar such that $|a|>1$. Then the $p \times m$ transfer matrix $\mathbf{G}(z)$, given by the right MFD of (25) is also BR. Furthermore, if $a$ is such that (29) holds, then the (determinantal) degree of the denominator matrix $\mathbf{D}_{1}(z)$ of $\mathbf{G}(z)$ is lower than that of $\mathbf{D}(z)$ by at least $m$.

Proof: It only remains to prove that $\mathbf{G}(z)$ is BR. To this end, we first show that $\mathbf{G}(z)$ is stable, and then cstablish that it satisfies the inequality:

$$
\mathbf{G}^{\dagger}\left(e^{j \omega}\right) \mathbf{G}\left(e^{j \omega}\right) \leqslant \mathbf{I}_{m}
$$

for all $\omega$. Accordingly, first consider the determinantal zeros of $\mathbf{D}_{1}(z)$ which are solutions of

$$
\operatorname{det}\left[\mathbf{I}_{m}-\kappa^{t} \mathbf{H}(z)\right]=0 .
$$

Thus if $z_{0}$ is such a zero, then there exists a non-null vector $v$ such that

$$
\left[\mathbf{I}_{m}-\boldsymbol{\kappa}^{t} \mathbf{H}\left(z_{0}\right)\right] \mathbf{v}=\mathbf{0} .
$$

In order to show that $\mathbf{G}(z)$ is stable, it is sufficient ${ }^{3}$ to show that the solutions $\mathrm{z}_{0}$ are strictly inside the unit circle. Let us assume the contrary, i.e., $\left|z_{0}\right| \geqslant 1$, and obtain a conflict. Equation (35) implies

$$
\mathbf{v}^{\dagger} \mathbf{v}=\mathbf{v}^{\dagger} \mathbf{H}^{\dagger}\left(z_{0}\right) \kappa \kappa^{t} \mathbf{H}\left(z_{0}\right) \mathbf{v}
$$

Now, if $\mathbf{H}\left(z_{0}\right) \mathbf{v}$ were to be null, it would imply, by (35), that $\mathbf{v}$ itself is null. Assuming therefore that $\mathbf{H}\left(z_{0}\right) \mathbf{v}$ is non-null, by (32) we have

$$
\mathbf{v}^{\dagger} \mathbf{H}^{\dagger}\left(z_{0}\right) \kappa \kappa^{t} \mathbf{H}\left(z_{0}\right) \mathbf{v}<\mathbf{v}^{\dagger} \mathbf{H}^{\dagger}\left(z_{0}\right) \mathbf{H}\left(z_{0}\right) \mathbf{v} .
$$

\footnotetext{
${ }^{3}$ This is also necessary if the MFD for $\mathbf{G}(z)$ is irreducible [13]
} 
Notice that the inequality in (37) is strict because, the inequalities of (32) are strict, which in turn is true because, by the theorem statement, $\mathbf{H}(z)$ is not memoryless in any direction. Equations (36) and (37) imply that

$$
\mathbf{v}^{\dagger} \mathbf{v}<\mathbf{v}^{\dagger} \mathbf{H}^{\dagger}\left(z_{0}\right) \mathbf{H}\left(z_{0}\right) \mathbf{v} .
$$

But by $\mathrm{BR}$ property, $\mathbf{H}(z)$ already satisfies the inequality (23) for $|z| \geqslant 1$. This leads to the impossible inequality:

$$
\mathbf{v}^{\dagger} \mathbf{v}<\mathbf{v}^{\dagger} \mathbf{v}
$$

This shows that all determinantal zeros of $\mathbf{D}_{1}(z)$ are strictly inside the unit circle, hence $\mathbf{G}(z)$ is stable.

In order to prove the inequality (33), it suffices to show that:

$$
\mathbf{G}_{2}^{\dagger}\left(e^{j \omega}\right) \mathbf{G}_{2}\left(e^{j \omega}\right) \leqslant \mathbf{I}_{m}
$$

where

$$
\begin{aligned}
\mathbf{G}_{2}(z)= & \mathbf{N}_{2}(z) \mathbf{D}_{2}^{-1}(z) \\
= & \left(\mathbf{I}_{p}-\kappa \kappa^{t}\right)^{-1 / 2}[\mathbf{H}(z)-\kappa] \\
& \cdot\left[\mathbf{I}_{m}-\kappa^{t} \mathbf{H}(z)\right]^{-1}\left(\mathbf{I}_{m}-\kappa^{t} \kappa\right)^{1 / 2}
\end{aligned}
$$

with $\kappa$ satisfying (32). It is easily verified that $\mathbf{N}_{2}(z)$ and $\mathbf{D}_{2}(z)$ are related by

$$
\begin{aligned}
& \mathbf{N}(z)=\mathscr{C} \mathbf{D}_{2}(z)+\mathscr{D} \mathbf{N}_{2}(z) \\
& \mathbf{D}(z)=\mathscr{A} \mathbf{D}_{2}(z)+\mathscr{B} \mathbf{N}_{2}(z)
\end{aligned}
$$

where the parameters $\mathscr{A}, \mathscr{B}, \mathscr{C}, \mathscr{D}$ are given by

$$
\begin{array}{ll}
\mathscr{A}=\left(\mathbf{I}_{m}-\kappa^{t} \kappa\right)^{-t / 2}, & \mathscr{B}=\kappa^{t}\left(\mathbf{I}_{p}-\kappa \kappa^{t}\right)^{-t / 2} \\
\mathscr{C}=\kappa\left(\mathbf{I}_{m}-\kappa^{t} \kappa\right)^{-t / 2}, & \mathscr{D}=\left(\mathbf{I}_{p}-\kappa \kappa^{t}\right)^{-t / 2} .
\end{array}
$$

It can further be verified that the parameters $\mathscr{A}, \mathscr{B}, \mathscr{C}, \mathscr{D}$ satisfy

$$
\begin{aligned}
\mathscr{A}^{t} \mathscr{A}-\mathscr{C}^{t} \mathscr{C} & =\mathbf{I}_{m}, \\
\mathscr{D}^{t} \mathscr{D}-\mathscr{B}^{t} \mathscr{B} & =\mathbf{I}_{p}, \\
\mathscr{A}^{t} \mathscr{B} & =\mathscr{C}^{t} \mathscr{D} .
\end{aligned}
$$

By making use of (42), (43), (45), and the fact that $\mathrm{H}(z)$ already satisfies (5), we can verify the following inequality:

$$
\mathbf{N}_{2}^{\dagger}\left(e^{j \omega}\right) \mathbf{N}_{2}\left(e^{j \omega}\right) \leqslant \mathbf{D}_{2}^{\dagger}\left(e^{j \omega}\right) \mathbf{D}_{2}\left(e^{j \omega}\right)
$$

which proves $(40)$. This then completes the proof of the theorem.

As a corollary, note that if $\mathbf{H}(z)$ is actually $L B R$, and if $|a|>1$ then $\mathbf{G}(z)$ is also LBR. This is because, (5) now becomes an equality, hence (46) becomes an equality, hence (40) becomes an equality. Consequently, (33) becomes an equality, because equality (14) holds on the unit circle for any $a$.

\section{Network Interpretation of the Theorem}

Fig. 5 shows a circuit-interpretation of the above theorem. The parameters $\mathscr{A}, \mathscr{B}, \mathscr{C}, \mathscr{D}$, can be interpreted as the chain parameters of a matrix digital two-pair. Such. a two-pair is a generalization of the two-pair described by (7), except that the quantities involved are now matrices of

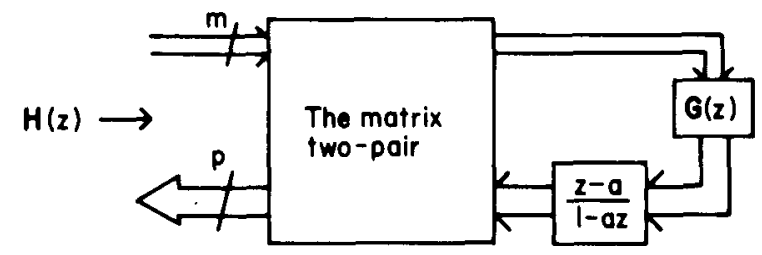

Fig. 5. Circuit implementation of (30).

appropriate dimensions. (Note that, the two-pair is constant i.e., not a function of $z$ ). The equivalent transferparameter description is as in (8) and the two descriptions are related as

$$
\begin{aligned}
& \mathbf{T}_{11}=\mathscr{C} \mathscr{A}^{-1}, \\
& \mathbf{T}_{12}=\mathscr{D}-\mathscr{C} \mathscr{A}^{-1} \mathscr{B}, \\
& \mathbf{T}_{21}=\mathscr{A}^{-1}, \\
& \mathbf{T}_{22}=-\mathscr{A}^{-1} \mathscr{D} .
\end{aligned}
$$

Now, with the chain parameters as in (44), it can be shown that the transfer matrix of the two-pair takes the following form $\tau=\left[\mathbf{T}_{i j}\right]$ where

$$
\tau=\left(\begin{array}{cc}
\kappa & \left(\mathbf{I}_{p}-\kappa \kappa^{t}\right)^{1 / 2} \\
\left(\mathbf{I}_{m}-\kappa^{t} \boldsymbol{\kappa}\right)^{t / 2} & -\left(\mathbf{I}_{m}-\kappa^{t} \boldsymbol{\kappa}\right)^{t / 2} \boldsymbol{\kappa}^{t}\left(\mathbf{I}_{p}-\kappa \kappa^{t}\right)^{-t / 2}
\end{array}\right) .
$$

It is easily verified that this matrix is orthogonal, i.e., $\tau^{t} \tau=\mathbf{I}_{m+p}$. In other words, the process of obtaining the remainder $\mathrm{BR}$ matrix $\mathbf{G}(z)$ from the $\mathrm{BR}$ matrix $\mathbf{H}(z)$ by using (25) can be looked upon as an LBR (matrix) two-pair extraction. The details of the circuit of Fig. 5 are as shown in Fig. 6, and the resemblance to the normalized scalar lattice (Fig. 4) is clear. Indeed if $p=m=1$, then the results of this section reduce to those of Section II.

Next, in order to avoid delay-free loops in the structure of Fig. 6, it becomes necessary to pick $a$ to be $\infty$. Equation (25) then becomes,

$$
\begin{aligned}
z^{-1} \mathbf{G}(z)=\left(\mathbf{I}_{p}-\kappa \kappa^{t}\right)^{-1 / 2}[\mathbf{H}(z)-\kappa] \\
\cdot\left[\mathbf{I}_{m}-\kappa^{t} \mathbf{H}(z)\right]^{-1}\left(\mathbf{I}_{m}-\kappa^{t} \kappa\right)^{1 / 2} .
\end{aligned}
$$

This results in the structure of Fig. 7, which is a generalization of Fig. 4. The structure of Fig. 7, which is a special case of that in Fig. 6, has already been reported in [26], based on a different approach. Finally, if the BR function $\mathbf{H}(z)$ is actually LBR, then the condition of (29) automatically holds. In particular, with the choice $a=\infty$, we can synthesize a $p \times m$ LBR transfer matrix in the form of Fig. 8, where each two-pair building block is as in Fig. 7, provided that (32) holds with strict inequality, at each stage of LBR two-pair extraction. Note that each building block is characterized by a $(p+m) \times(p+m)$ orthogonal transfer matrix. The termination matrix $\mathbf{R}$ in Fig. 8 is such that $\mathbf{R}^{t} \mathbf{R}=\mathbf{I}_{m}$. A special case of these structures with $p=2$, $m=1$ has already been reported in the literature, even though their derivations followed a different approach [8], 


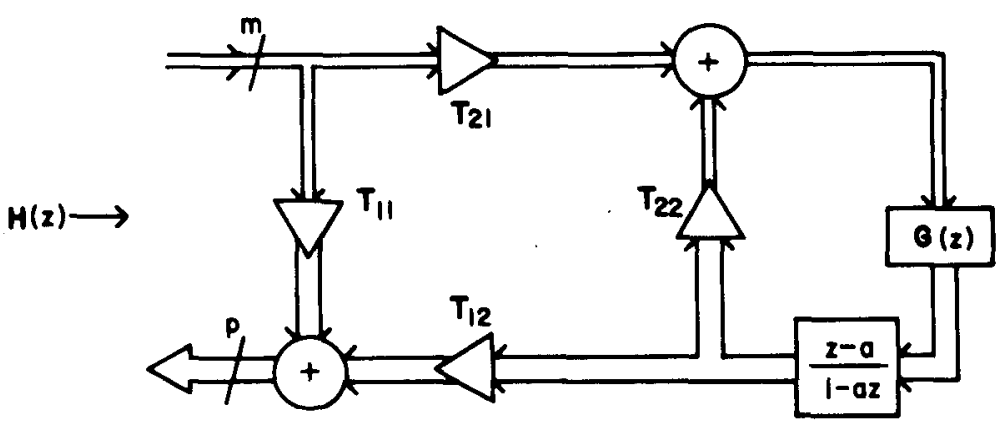

Fig. 6. Details of the circuit of Fig. 5. $\mathbf{T}_{i j}$ are as in (47).

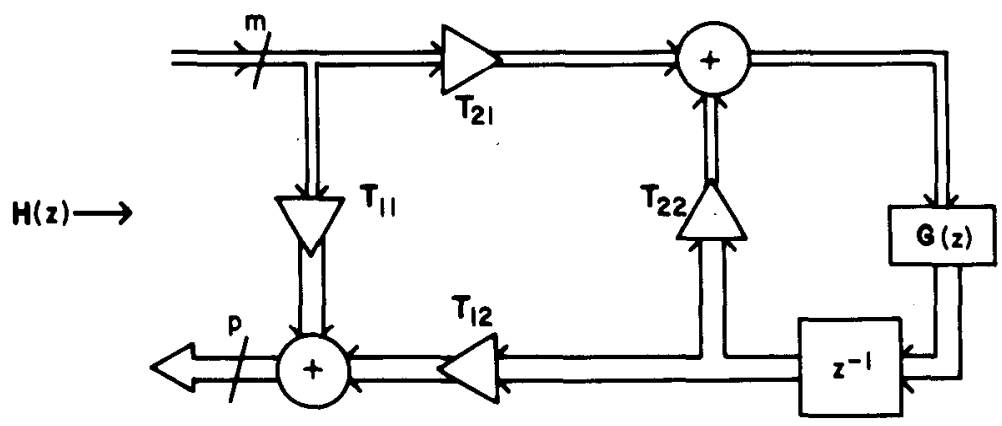

Fig. 7. Special case, where $a=\infty$.

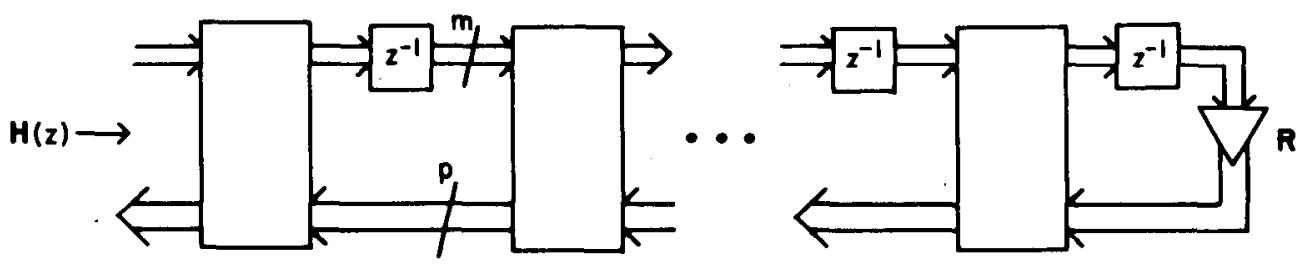

Fig. 8. The cascaded lattice structure for a $p \times m$ LBR transfer matrix H(z). Each two-pair has the form shown in Fig. 7.

[9]. Note that, because of the delay-units separating successive two-pairs in Fig. 8, the structures are pipelineable, which is a desirable property in an actual implementation. Finally note that, the only building blocks in Fig. 8 are orthogonal matrices, which can be implemented in terms of several planar rotations [14]. This can lead to a cordicprocessor based implementation.

\section{Realization of Arbitrary Stable Transfer Matrices}

Now, given an arbitrary $k \times m$ transfer matrix $\mathbf{H}_{1}(z)$ that is not necessarily all pass:

$$
\mathbf{H}_{1}(z)=\mathbf{A}(z) \mathbf{D}^{-1}(z)
$$

we can still apply the above procedure for synthesizing it in the form of a cascaded lattice. For this, let us assume that $\mathbf{H}_{1}(z)$ is scaled so that the inequality analogous to (5) holds. Then we can embed $\mathbf{H}_{1}(z)$ into an "all-pass" matrix $\mathbf{H}(z)$ of the form of (20), and then synthesize $\mathbf{H}(z)$ [26].

An important application of these structures is in the synthesis of low sensitivity digital filters. Indeed it can be shown, based on the "structural passivity" argument [21], that transfer functions synthesized in this manner have low passband sensitivity. For example, a scalar transfer function can be synthesized as a cascaded lattice simply by embedding it into a $2 \times 1$ LBR matrix, synthesizing the LBR matrix, and ignoring the unwanted output. The resulting structure has low sensitivity, and leads to pipelineable, cordic-processor based implementations [9].

\section{Application to Stability-Tests}

It is well known that Jury's method for testing the stability of discrete-time scalar linear systems [15], [16] is directly related to the synthesis of an all-pass function in the form of a cascaded lattice. Let us first briefly review this relation, so that we can extend these ideas for the case of $p \times m$ transfer matrices. Given a transfer function $H_{1}(z)=A(z) / D(z)$ with no common factors between $A(z)$. and $D(z)$, an allpass function $H(z)$ is first formed:

$$
H(z)=z^{-N} D\left(z^{-1}\right) / D(z)
$$

where $N$ is the degree of $D(z)$. This all-pass function is LBR if and only if it is stable. (If there is a common factor between the numerator and denominator of $H(z)$, it automatically gets detected during the test [26]. We therefore assume that there is no such factor. In other words, $H(z)$ is assumed to be irreducible.) Therefore, checking for the stability of $H_{1}(z)$ is equivalent to checking if $H(z)$ is LBR. To avoid trivialities, assume that $H(z)$ is not a constant 
function. From the discussions in Section II we know that if $I(z)$ is LBR then

$$
|k|<1
$$

where $k$ is given by (18):

$$
k=H(\infty) \text {. }
$$

Thus if (52) is not satisfied, $H(z)$ is unstable, and the test terminates. If (52) is satisfied, then the following is true:

$$
G(z) \text { is } L B R \text { if and only if } H(z) \text { is } L B R
$$

where $G(z)$ is the reduced order remainder shown in Fig. 3 , and given by

$$
z^{-1} G(z)=\frac{H(z)-k}{1-k H(z)} .
$$

From the arguments of Section II, it is clear that $G(z)$ should be LBR if $H(z)$ is. The converse in (53) can be seen by inverting (54) (i.e., writing $H(z)$ in terms of $z^{-1} G(z)$ ) and repeating these arguments. As a result, it suffices to test for the stability of $G(z)$.

The general test procedure can now be described as follows: Given the $N$ th-order transfer function $H_{1}(z)$, we form the $N$ th-order all-pass function $G_{N}(z)=H(z)$ as in (51), and compute the coefficient $k_{N}=G_{N}\left(a_{N}\right)$ where $a_{N}$ is any point outside the unit circle. We then generate the lower order all-pass function $G_{N-1}$ according to

$$
z^{-1} G_{N-1}=\frac{G_{N}(z)-k_{N}}{1-k_{N} G_{N}(z)}
$$

and repeat the same process with $G_{N-1}(z)$. Thus we have generated the sequence of all-pass functions of decreasing order

$$
G_{N}(z), G_{N-1}(z), \cdots G_{1}(z), G_{0}(z)
$$

and the series of lattice coefficients

$$
k_{N}, k_{N-1}, \cdots k_{1}, k_{0}
$$

where

$$
k_{m}=G_{m}\left(a_{m}\right) .
$$

The original transfer function is stable if and only if $\left|k_{m}\right|<1$ for all $m$. Notice that it is not necessary to choose $a_{m}=\infty$. Even though there are delay free loops in the structure of Fig. 2 for $a_{m} \neq \infty$, this does not matter for the purposes of the test. Such flexibility of the choice of $a_{m}$ is sometimes useful, as demonstrated in the following example.

A Numerical Example: Let us assume that the transfer function to be tested for stability has denominator

$$
D(z)=1+\frac{7}{6} z^{-1}-\frac{17}{6} z^{-2}+z^{-3} \text {. }
$$

The third-order all-pass function to be formed is, therefore,

$$
G_{3}(z)=\frac{1-\frac{17}{6} z^{-1}+\frac{7}{6} z^{-2}+z^{-3}}{1+\frac{7}{6} z^{-1}-\frac{17}{6} z^{-2}+z^{-3}} .
$$

When $a_{3}=\infty$ we get $k_{3}=G_{3}(\infty)=1$. Clearly (52) is not satisfied, and hence the system is unstable. However, if we wish to proceed further with the generation of all the $k_{m}$-parameters, so that the lattice structure can be completely generated, we encounter a difficulty. Thus referring to (54), if $k=1$, the right hand side becomes identically equal to a constant, and the quantity $G(z)$ is not meaningful anymore. Such a singularity situation can be avoided simply by taking $a_{3}$ to be some other point outside the unit circle. (Since $G_{3}(z)$ is a nonconstant rational function, there exist an infinite number of points in the $z$-plane such that its magnitude is different from unity). For example, let $a_{3}=3$ then $k_{3}=G_{3}(3)=1 / 5$. The remainder allpass function $G_{2}(z)$ can now be computed as

$$
G_{2}(z)=\frac{-3+\frac{5}{2} z^{-1}+z^{-2}}{1+\frac{5}{2} z^{-1}-3 z^{-2}} .
$$

Let us now proceed to the next iteration. Thus let $a_{2}=\infty$ and compute $k_{2}=G_{2}(\infty)=-3$. The remainder allpass function $G_{1}$ is now

$$
G_{1}(z)=\frac{\frac{-5}{4}+z^{-1}}{1-\frac{5}{4} z^{-1}} .
$$

Proceeding further with the next iteration, let us pick $a_{1}=\infty$ so that $k_{1}=G_{1}(\infty)=-5 / 4$. We thus have the set of lattice coefficients

$$
k_{3}=1 / 5, \quad k_{2}=-3, \quad k_{1}=-5 / 4 .
$$

Clearly, the system is not stable. It can further be shown that there is one unstable pole.

It remains to explain why it is sometimes of interest to compute all the lattice coefficients and hence complete the lattice generation, even if the system is unstable. This is necessary when we wish to compute the number of unstable poles of the system. In general, if one wishes to enumerate the zeros of a polynomial outside a circle of a certain radius, the completion of the lattice-generation process is essential. Such counting procedures are based on judicious applications of Rouche's theorem of complex variable theory, and some details can be found in [24], [25].

The generalized version of Richards' theorem, given in Section III can now be employed to obtain similar developments for the $p \times m$ transfer matrix case. Let us assume $a=\infty$, so that (49) is relevant. Let us consider a $p \times m$ all-pass transfer matrix $\mathbf{H}(z)$, i.e., a matrix of the form (20) satisfying

$$
\mathbf{H}^{\dagger}\left(e^{j \omega}\right) \mathbf{H}\left(e^{j \omega}\right)=\mathbf{I}_{m}
$$

for all $\omega$. Once (62) is satisfied, the terms "stable" and "LBR" are equivalent. Assume, as in the scalar case, that $\mathbf{H}(z)$ is given as an irreducible ${ }^{4}$ right MFD, as in (20). Then $\mathbf{H}(z)$ is LBR if and only if all the determinantal zeros of $\mathbf{D}(z)$ are strictly inside the unit circle. We know that, if $\mathbf{H}(z)$ is indeed LBR, then $\kappa$ defined by (27):

$$
\kappa=\mathbf{H}(\infty)
$$

satisfies

$$
\kappa^{t} \kappa \leqslant \mathbf{I}_{m}
$$

\footnotetext{
${ }^{4}$ That is $\mathbf{N}(z)$ and $\mathbf{D}(z)$ are right coprime [13]
} 
Thus if (64) does not hold, the test terminates. If (64) holds but not with strict inequality, this implies that $\mathbf{H}(z)$ is memoryless in some direction [26], as explained in Section III. The memoryless direction can be decoupled and removed [26], and we can thus assume the inequality of (64) to be strict. Let us therefore assume that, if (64) holds, it actually holds with strict inequality. Then the following is true:

$$
\mathbf{G}(z) \text { is } L B R \text { if and only if } \mathbf{H}(z) \text { is } L B R \text {. }
$$

The "if" part in (65) is clear, from Section III. In order to see the "only if" part, we have to show that $H(z)$ is I.BR (i.e., stable) if $\mathbf{G}(z)$ is LBR. Recall from (43), that the determinantal zeros of $\mathbf{D}(z)$ are precisely the solutions of

$$
\operatorname{det}\left[\mathscr{A}+z^{-1} \mathscr{B} \mathbf{G}(z)\right]=0 .
$$

As $\mathscr{A}$ (equation (44)) is nonsingular, we can rewrite (66) with the help of (47) as

$$
\operatorname{det}\left[\mathbf{I}_{m}-z^{-1} \mathbf{T}_{22} \mathbf{G}(z)\right]=0 .
$$

As $\tau$ in (48) is orthogonal, it is easy to verify that $\mathbf{T}_{22}$ satisfies

$$
\mathbf{T}_{22}^{t} \mathbf{T}_{22}<\mathbf{I}_{p}
$$

Moreover, $\mathbf{G}(z)$, being LBR, satisfies an inequality analogous to (23), for $|z| \geqslant 1$. By arguments similar to those following (34), it is immediately clear that $\mathbf{H}(z)$ is stable, and hence LBR.

The stability-test procedure is therefore clear: compute $\kappa$ and check for the inequality (64). If this is not satisfied, this implies instability of $\mathbf{H}(z)$, and further testing is discontinued. If strict inequality holds, then repeat the process with the reduced-degree remainder $\mathbf{G}(z)$. If the inequality is not strict, decouple the appropriate memoryless direction [26], so that the inequality becomes strict, and then proceed further. Clearly, the procedure terminates after a finite number of steps. Checking for condition (64) is equivalent to checking for the positive semi-definiteness of $\mathbf{I}_{m}-\boldsymbol{\kappa}^{t} \boldsymbol{\kappa}$.

\section{Testing Arbitrary Transfer Matrices}

If the given transfer matrix is not all pass, we can proceed as follows: ignore the numerator polynomial, and define a transfer matrix $\mathbf{H}_{1}(z)$ as in (50) of Section III., with $\mathbf{A}(z)=\mathbf{I}_{m}$. Then construct an all-pass matrix $\mathbf{H}(z)$ of the form (20) as in Section III, by defining $\mathbf{N}(z)$ "appropriately". This all-pass function can now be tested for stability. The construction of an appropriate $\mathbf{N}(z)$ such that $(20)$ is all-pass for a given $D(z)$ itself could be a nontrivial task; note that this should be done in such a way that the MFD in (20) is irreducible. The details of this are not within the scope of this paper.

\section{Summary and Concluding Remarks}

The $(p+m) \times(p+m)$ lattice structure developed in Section III leads to generalizations of the scalar lattice in a number of ways. First, it gives rise to a generalization of the synthesis of a scalar all-pass function. Second, the building blocks can be normalized so that they are lossless (or orthogonal), just as in the scalar case. Third, the synthesis of a $p \times m$ all-pass matrix can be directly related to a generalized version of Jury's stability test.

In the statement of generalized Richards' Theorem of Section III, we did not assume that the MFD for $\mathbf{H}(z)$ is irreducible. As a result, the cascaded lattice structure of Fig. 8 can be obtained even for non-minimal MFD's. However, it can be shown that [26], any common right divisor $\mathbf{R}(z)$ between the matrices $\mathbf{N}(z)$ and $\mathbf{D}(z)$ is also a right divisor in the MFD for $\mathbf{G}(z)$. Consequently, the greatest common right divisor (GCRD) propagates "down the line" during the lattice synthesis. This is analogous to the situation of scalar allpass synthesis, where any common factor between the numerator and denominator gets detected by a prematured termination of the synthesis process. Indeed, the results of Section III can be related to a generalization of Euclid's algorithm for extracting the GCRD. Finally, we feel that the results of Section III can be related to the theory of "matrix polynomials that are orthogonal on the unit circle [17]," but the details are beyond the scope of this paper.

It should be pointed out that, the condition of (29) is not necessary for degree reduction. Indeed, if the matrix on the left-hand side of (29) is singular, this is sufficient to bring about a reduction in determinantal degree of the denominator. However, the reduction is then not as large as $m$.

\section{ACKNOWLEDGMENT}

The authors are thankful to Dr. Y. Neuvo of the Tampere University of Technology, for useful discussions.

\section{REFERENCES}

[1]. G. C. Temes and J. W. LaPatra, Circuit Synthesis and Design New York: McGraw-Hill, 1977.

[2] L. Weinberg, Network Analysis and Synthesis. New York: McGraw-Hill, 1962.

[3] J. D. Markel and A. H. Gray, Jr., "Roundoff noise characteristics of a class of orthogonal polynomial structures," IEEE Trans. Acoust., Speech, Signal Processing, vol. ASSP-23, pp. 473-485, Oct. 1975.

[4] A. H. Gray, Jr., and J. D. Markel, "A normalized digital filter structure," IEEE Trans. Acoust., Speech, Signal Processing, vol. ASSP-23, pp. 268-277, June 1975.

[5] J. D. Markel and A. H. Gray, Linear Prediction of Speech. New York: Springer Verlag, 1976.

[6] S. K. Mitra, P. S. Kamat, and D. C. Huey, "Cascaded lattice realization of digital filters," Int. J. Circuit Theory Appl., vol 5, pp. $3-11,1977$.

[7] P. P. Vaidyanathan, "A General theory and synthesis procedure for low sensitivity digital filter structures," Ph.D. dissertation, Univ. of California, Santa Barbara.

[8] D. Henrot and C. T. Mullis, "A modular and orthogonal digital filter structure for parallel processing," in IEEE Int. Conf. on Acoustics, Speech, and Signal Processing, pp. 623-626, Boston, MA, Apr. 1983.

[9] S. K. Rao and T. Kailath, "Pipelined orthogonal digital lattice filters," in IEEE Int. Conf. on Acoustics, Speech, and Signal Processing, pp. 11.10.1-11.10.4, San Diego, CA, Apr. 1984

[10] B. D. O Anderson, Network Analysis and Synthesis. Englewood Cliffs, NJ: Prentice Hall, 1973.

[11] A. V. Oppenheim and R. W. Schafer, Digital Signal Processing. Englewood Cliffs, NJ: Prentice Hall, 1975.

[12] A. H. Gray, Jr., "Passive Cascaded lattice digital filters," IEEE Trans. Circuits Syst., vol. CAS-27, pp. 337-344, May 1980.

[13] T. Kailath, Linear Systems. Englewood Cliffs, NJ: Prentice Hall, 1980.

[14] F. D. Murnaghan, The Unitary and Rotation Groups. Washington, DC: Spartan, 1962.

[15] E. I. Jury, Theory and Application of the z-Transform Method. New York: Wiley, 1964. 
[16] A. Antoniou, Digital Filters: Analysis and Design. New York: McGraw Hill, 1979.

[17] P. Delsarte, Y. V. Genin, and Y. G. Kamp, "Orthogonal polynomial matrices on the unit circle," IEEE Trans. Circuits Syst., vol. CAS-25, pp. 149-160, Mar. 1978.

[18] H. J. Carlin, "The scattering matrix in network theory," IRE Truns. Circuit Theory, vol. 3, pp. 88-97, June 1956.

[19] D. C. Youla, "On the factorization of rational matrices," IRE Trans. Inform. Theory, vol. 7, pp. 172-189, July 1961.

[20] C. A. Desoer and J. D. Schulman, "Zeros and poles of matrix transfer functions and their dynamical interpretation," IEEE Trans. Circuits Syst., vol. CAS-21, pp. 3-8, Jan. 1974.

[21] P. P. Vaidyanathan and S. K. Mitra, "Low passband sensitivity digital filters: a generalized viewpoint and synthesis procedures," Proc. IEEE, vol. 72, pp. 404-423, Apr. 1984.

[22] P. P. Vaidyanathan, "A unified approach to orthogonal digital filters and wave digital filters, based on LBR two-pair extraction," IEEE Trans. Circuits Syst., vol. 32, pp. 673-686, July 1985.

[23] Ph. Delsarte, Y. Genin, and Y. Kamp, "On the role of the Nevanlinna-Pick problem in circuit and system theory," Int. J. Circuit Theory Appl., vol. 9, pp. 177-187, 1981.

[24] M. Marden, "Gcomctry of polynomials," Amer. Math. Soc., Rhode Island, 1966

[25] D. Williamson, "A note on stability and lattice filter realizations," IEEE Trans. Acoust. Speech, Signal Processing vol. ASSP-31, pp. $770-772$, June 1983.

[26] P. P. Vaidyanathan and S. K. Mitra, "A general family of multivariable digital lattice filters," IEEE Trans. Circuits Syst., vol. CAS-32, pp. 1234-1245, Dec. 1985.

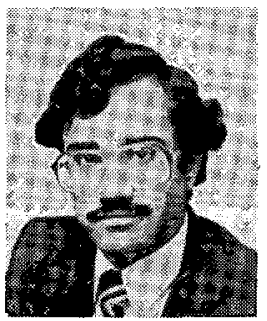

P. P. Vaidyanathan (''80-M'83) was born in Calcutta, India, on October 16, 1954. He received the B.Sc. (hons.) degree in physics, and B. Tech. and $M$. Tech. degrees in Radiophysics and electronics from the University of Calcutta, India, in 1974,1977 , and 1979, respectively, and the Ph.D. degree in electrical and computer engineering from the University of California, Santa Barbara, in 1982.

He was a Post-Doctoral fellow at the University of California, Santa Barbara, from September 1982 to February 1983. Since March 1983, he has been with the California Institute of Technology, Pasadena, as an Assistant Professor of Electrical Engineering. He was recipient of the award for Excellence in Teaching at the California Institute of Technology, for the year 1983-1984. His main research interests are in digital signal processing, linear systems and filter design. He served as the Vice-Chairman of the Technical
Program Committee for the 1983 IEEE International Symposium on Circuits and Systems. Dr. Vaidyanathan currently serves as an Associate Editor for the IEEE Transactions on Circuits and Systems."

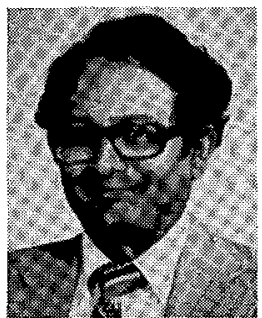

Sanjit K. Mitra (S'59-M'63-SM'69-F'74) received the B.S. (hons.) degree in physics from the Utkal University, India, in 1953, the M.Sc. (Tech.) degree in radio physics and electronics from the University of Calcutta, India, in 1956, the M.S. and Ph.D. degrees in electrical engineering from the University of California, Berkeley, in 1960 and 1962, respectively.

He was a member of the faculty of the Cornell University, Ithaca, NY, from 1962 to 1965 , and a member of Technical Staff of the Bell Laboratories from 1965 to 1967 . He joined the faculty of the University of California, Davis, in 1967 and transferred to the Santa Barbara (UCSB) campus in 1977 as a Professor of Electrical and Computer Engineering. From July 1979 to June 1982 he served as the Chairman of the Department of Electrical and Computer Engineering at UCSB. He is a consultant to the Lawrence Livermore National Laboratory, Livermore, CA, and the Los Alamos National Laboratory, Los Alamos, NM, and is the Consulting Editor for the Electrical/Computer Science and Engineering Series of the Van Nostrand-Reinhold Company, NY. He has also served as a consultant to Ampex Corporation, Fairchild Semiconductor, Siliconix, HDR Sciences, the U.S. Army, General Motors Delco Division and the World Bank. He has held visiting appointments at the Indian Institute of Technology, New Delhi; Kobe University, Japan; University of Erlangen-Nuernberg, West Germany, the Australian National University, Canberra; Tampere University of Technology, Tampere, Finland, and the Federal University of Rio de Janeiro, Brazil. He has published a number of papers in active and passive networks, and digital filters, and is the author of Analysis and Synthesis of Linear Active Networks and An Introduction to Digital and Analog Integrated Circuits and Applications, and Editor of Active Inductorless Filters, Co-Editor of Modern Filter Theory and Design (with G. C. Temes), Co-Editor of Two-Dimensional Digital Signal Processing (with M. P. Ekstrom). He holds two patents in active filters. He was an Associate Editor of the IEEE TrANSACTIONS ON Circuits AND Systems, and served on the editorial boards of the ProCEEDINGS OF THE IEEE and IEEE Press, and presently is on the editorial board of Circuits, Systems and Signal Processing. He has been a member of the Administrative Committee of the IEEE Circuits and Systems Society, Local Arrangements Chairman (1969), General Chairman (1974), and Program Chairman (1983) of the annual IEEE International Symposium on Circuits and Systems. He is the recipient of the 1973 F.E. Terman Award of the American Society of Engineening Education, a Visiting Professorship from the Japan Society for Promotion of Science in 1972, and Senior Fulbright Award for Brazil in 1984

Dr. Mitra is a Fellow of the American Association for the Advancement of Science and a member of the American Society for Engineering Education, Sigma Xi, and Eta Kappa Nu. 